\title{
Construction of a High-Density Genetic Map and Mapping of Major Flowering Time QTLs in Rapeseed (Brassica napus L.) Based on Whole- Genome Re-sequencing
}

\section{Xin Li}

Academy of Agricultural and Forestry Sciences of Qinghai University

\section{Dezhi Du}

Academy of Agricultural and Forestry Sciences of Qinghai University

\section{Qianru Ma}

Academy of Agricultural and Forestry Sciences of Qinghai University

\section{Linfang Li}

Academy of Agricultural and Forestry Sciences of Qinghai University

\section{Zhi Zhao}

Academy of Agricultural and Forestry Sciences of Qinghai University

Guoyong Tang

Academy of Agricultural and Forestry Sciences of Qinghai University

Haidong Liu

Academy of Agricultural and Forestry Sciences of Qinghai University

Lu Xiao ( $\nabla$ xlu2005@yahoo.com.cn )

Academy of Agricultural and Forestry Sciences of Qinghai University

\section{Research article}

Keywords: Spring Brassica napus L., Recombinant inbred lines, Flowering time, Environmentally stable and specific QTLs, BnaA02g12130D

Posted Date: January 13th, 2020

DOl: https://doi.org/10.21203/rs.2.20743/v1

License: (1) This work is licensed under a Creative Commons Attribution 4.0 International License. Read Full License 


\section{Abstract}

\section{Background}

Flowering time is an important trait impacting the performance of Brassica napus L. cultivars, but the interaction of all the loci controlling this trait in spring-type oilseed rape is not fully understood.

\section{Results}

In this study, we conducted a genome-wide quantitative trait locus (QTL) analysis of a recombinant inbred line (RIL) population composed of 215 lines and constructed a high-density linkage map with 4523 recombination bin markers and of 92,627 single-nucleotide polymorphisms (SNPs). After phenotypic measurements across four different fields environments were collected for 2 years, at least 27 flowering time QTLs were identified; these were distributed mainly on linkage groups (LGs) A02 (11 QTLs), A10 (1 QTL), C02 (12 QTLs), C05 (2 QTLs) and C08 (1 QTL). Of these QTLs, five major QTLs for flowering time were detected, and three of them, sqFT-A02-3, sqFT-A02-4, and sqFT-A10-1, were identified only in winter ecological conditions; thus, these QTLs were environment specific. The QTL cqFT-C02-2 was detected in all 3 spring environments, showing strong spring environmental specificity. The major QTL cqFT-C02-3, which is an environmentally stable QTL, was detected in all 4 environments and was expressed both in spring and winter ecological conditions. The winter ecological condition-specific major QTL sqFT-A02-4 was delimited to a region of approximately $216 \mathrm{~kb}$, between $6.302 \mathrm{Mb}$ and $6.518 \mathrm{Mb}$ on $\mathrm{A} 02$. In this region, the BnaA02g12130D/GSBRNA2T00090951001 gene was identified as a homologue of the Arabidopsis thaliana FLOWERING LOCUS T (FT) gene by a BLASTn analysis of the candidate region.

\section{Conclusions}

Five major novel QTLs for flowering time were discovered in spring Brassica napus: 1 environmentally stable QTL and 4 environment-specific QTLs. The major QTL sqFT-A02-4 was delimited to a candidate region of approximately $216 \mathrm{~kb}$, and $B n a A 02 g 12130 \mathrm{D}$ was determined to be a potential gene

\section{Background}

Brassica napus ( $B$. napus, AACC, $2 n=38$ ) is one of the most important allotetraploid rapeseed species, and owing to its high seed oil and protein content, this species is grown mainly to provide vegetable oil for humans, animal fodder and biodiesel for industrial purposes [1]. In many geographical regions with long, cold winters, such as Canada, only spring-type rapeseed with early-flowering habits can be selected for growing to partly prevent rapeseed plants from suffering from winter injury, which helps maintain sustainable rapeseed development and the production exceptionally high-quality seeds [2]. In the southern part of China, the time for changing between rapeseed and cotton oil crops overlaps, and the farmland is largely idle during the winter, thus reducing the growing area of rapeseed [3]. The QinghaiTibet Plateau in China is planted mainly with spring-type $B$. napus. Due to the high altitude and cold climate of this plateau throughout the year, the cycle of suitable rapeseed is short; thus, it is necessary to 
plant early-maturing rapeseed varieties to harvest full grains and to improve rapeseed yield and quality [4]. Many studies have confirmed that there is a significant positive correlation between flowering time and maturity in rapeseed [5]. Therefore, the genetic study of flowering time is of great theoretical importance to the breeding of early-maturing varieties of rapeseed.

Flowering time is a complex quantitative trait in plants. In response to both endogenous and exogenous stimuli, including the photoperiod, temperature and hormones, flowering time is regulated by a complex network $[6,7]$. In the model plant species Arabidopsis thaliana (A. thaliana), six pathways involved in flowering time have been identified: those related to vernalization, photoperiod, gibberellic acid (GA), autonomy, the thermal clock and ageing [8-10]. Hundreds of genes in these six pathways participate in the regulation of $A$. thaliana flowering networks [11], and these genes are further integrated with other genes involved in flowering time [10]. FLOWERING LOCUS T $(F T)$ is an important integration factor and a key gene involved in floral regulation; $F T$ integrates the signals perceived in response to the photoperiod, vernalization and autonomy pathways and transmits them to downstream floral development genes, thereby promoting plant flowering [12]. The product of $F T$ expression is a flowering stimulant that is translocated from the leaf to the stem tip over long distances, ultimately causing flowering $[13,14]$. Many $F T$-like genes have been cloned, and their expression has been suggested to promote flowering in plants [15]. B. napus and $A$. thaliana are cruciferous plants and are closely related. In addition, knowledge of genes related to flowering in $B$. napus is largely based on $A$. thaliana via comparative genomics. B.napus BnFT (BnA2.FT, BnA7.FT.a, BnA7.FT.b, BnC2. FT, BnC6.FT and BnC6.FT.b) is homologous to the A. thaliana $F T$ gene, which is considered the key gene for determining flowering time in the photoperiod pathway [16].

Quantitative trait locus (QTL) analysis has been applied to identify potential candidate genes that control flowering time in $B$. napus [17]. Flowering time QTLs with large phenotypic effects have been mapped onto chromosomes A02, A09, A10, $\mathrm{C02}, \mathrm{C0} 3$ and $\mathrm{C0} 8$ in B. napus. Using a double-haploid (DH) population of 217 lines and a genetic linkage map with 495 markers, Liu et al. [18] detected a total of 48 QTLs related to flowering time. In addition, by performing genome-wide association mapping of flowering time with a $60 \mathrm{~K}$ single-nucleotide polymorphism (SNP) array for a diversity panel comprising 523 inbred rapeseed lines, Xu et al. [19] identified 35 flowering time QTLs. Li et al. [20] used a DH population of 348 lines and a genetic linkage map with SNP markers and detected a total of 55 QTLs related to flowering time, which explained $2.99-25.96 \%$ of the phenotypic variation.

The development of high-throughput sequencing technology has increased the precision of species research, and the use of modern molecular methods has become a new trend in QTL research. Highthroughput sequencing technology provides new opportunities and possibilities for crop genetics and breeding [21, 22]. Whole-genome re-sequencing (WGRS) is a powerful tool to identify various types of genetic variation, including SNPs, insertions/deletions (Indels), structure variation (SV), and copy number variation (CNV) [23]. The determination of these variations has been used in QTL mapping and gene cloning $[24,25]$. Compared with previous methods, this mapping method can significantly improve the efficiency of QTL mapping and marker development [26]. 
In this study, we constructed a high-density linkage map by re-sequencing recombinant inbred lines (RILs) comprising 215 individuals that were developed from a cross between spring-type No. 3379 with an earlyflowering phenotype and from a cross between spring-type No. 2839 with a markedly late-flowering phenotype. The parents and RIL population were planted in three spring and winter ecological environments, and the flowering times of the panel accessions were investigated. The objectives of this study were to (1) evaluate the genetic variation and identify environmentally stable and environmentspecific QTLs for flowering time based on the high-density genetic map in different ecological conditions, (2) delimit the winter ecological conditions-specific major QTL sqFT-A02-4 to a target region, and (3) select candidate genes associated with flowering time within the sqFT-A02-4 target region. The results should provide us with a better understanding of the genetic regulation of early flowering.

\section{Results}

\section{Phenotypic performance of the parents and the F1 and RIL population}

T tests and analyses of variance (ANOVAs) were used to compare the differences in flowering time between the parents and RIL population in four environments (Table 1). Significant differences between the parents were detected in the four environments. The average flowering time of the early-flowering parent No. 3379 in the four environments was 28.92 days, while that of the late-flowering parent No. 2839 reached 73.75 days; specifically in the winter ecological condition, this parent required 112.33 days to flower. The F1 plants flowered 11.62 days later than did No. 3379 and 33.21 days earlier than did No. 2839 (Additional file 1: Fig. S1), suggesting that the major early-flowering locus in No. 3379 might be dominant. The flowering time of the RILs exhibited continuous variation, which approximated a normal distribution, implying that the early flowering time of No. 3379 was quantitatively inherited (Additional file 1: Fig. S1). The flowering time of the RIL population showed a different distribution in the winter and spring ecological environments. The flowering time ranged from 34.67 to 60 days in the three spring ecological environments, while the flowering time in the winter ecological environment ranged from 21.67 to 108.67 days (Additional file 1: Fig. S1).

The ANOVA revealed highly significant differences $(P<0.001)$ caused by the genotype $(G)$, environment $(E)$ and the genotype $\times$ environment $(G \times E)$ interaction (Additional file 2: Table S1), which indicated that the flowering time was significantly influenced by both genetic and environmental factors. The broadsense heritability $\left(h^{2}\right)$ of the RIL population in the four environments was $80.01 \%$, demonstrating that QTLs controlling flowering time have a large effect on reducing the days to flowering. Due to the diversity in temperature and day length between the spring and winter ecological conditions, the flowering time between the winter ecological conditions and the three spring environments was relatively weakly correlated $(r=0.48-0.50, P<0.01)$ (Additional file 3: Table S2). Therefore, these results suggested that flowering time is a typical quantitative trait controlled by multiple genes and that it is greatly affected by a variety of environmental conditions. 
High-throughput sequencing generated $81,926,866,85,633,867$ and $1,068,590,738$ clean reads from No. 3379, No. 2839 and the 215 RILs, respectively, and the Q30 (base recognition error at the 0.001 level) percentages were $92.52 \%, 92.64 \%$ and $93.01 \%$, respectively. After aligning the clean data to the reference genome, the sequencing depth and genome coverage of each sample were obtained. The average depth of the two parents (No. 3379 and No. 2839) was 15x and 18x, respectively, and that of the RILs was approximately $2 \times$. The $1 \times$ genome coverage of parents No. 3379 and No. 2839 was $88.15 \%$ and $92.43 \%$, respectively, and that of the RILs was approximately $52.77 \%$. On the basis of the alignment results of the clean data to the reference genome, GATK software was used for SNP calling. A total of $1,151,864$ SNPs were detected between parents No. 3379 and No. 2839. After filtering, a total of 727,706 homozygous SNPs were identified for further analysis. After the filling and correction of SNPs, each bin was divided according to the recombination of the offspring. A total of 4674 bins were used as markers for constructing a high-density linkage map. A total of 4523 bin markers were ultimately mapped to the linkage groups (LGs), spanning a total length of $2126.46 \mathrm{cM}$, and the average distance was $0.47 \mathrm{cM}$ between adjacent bin markers (Additional file 4: Table S3, Additional file 5: Fig. S2). Of these bins, 2218 (49.0\%) were mapped to the A subgenome, with an average distance of $0.52 \mathrm{cM}$, and $2305(51.0 \%)$ bins were localized to the $C$ subgenome, with an average distance of $0.42 \mathrm{cM}$. A02 was the largest $L G$, which covered $190.22 \mathrm{cM}$ and contained 263 bin markers, and C05 was the smallest LG, which spanned 52.56 cM and contained 141 bin markers. In addition, LG C02 contained 410 bin markers showing high marker polymorphism. These results indicate that all the bin markers were thoroughly distributed throughout the genome, and approximately $98.79 \%$ of the intervals between adjacent markers were less than $5 \mathrm{cM}$.

In addition, to evaluate the quality of the genetic map, the collinearity between the genetic map and the $B$. napus reference genome was assessed. All bin markers were mapped to the $B$. napus reference genome. As shown in Additional file 6: Table S4 and in Additional file 7: Fig. S3, the Spearman correlation coefficients between the genetic map and physical map exceeded 0.9 for 17 chromosomes, excluding the coefficients corresponding to $\mathrm{A} 10$ (0.875) and C07 (0.871). The LGs thus have high levels of genetic collinearity with the physical map (Fig. 1).

\section{QTL analysis of flowering time and identification of environmentally stable and environment-specific QTLS}

The high-density genetic linkage map combined with the phenotypic data was used to detect QTLs for flowering time. A total of 27 QTLs associated with flowering time were identified in the RIL population (Table 2). These QTLs were distributed mainly on LGs A02 (11 QTLs), A10 (1 QTL), C02 (12 QTLs), C05 (2 QTLs) and C08 (1 QTL) and individually explained $3.25 \%$ to $23.78 \%$ of the phenotypic variance. The additive effect (from -0.79 to -9.02 days) of all the QTLs detected in the present study are negative, implying that parent No. 3379 contributed alleles for a shorter flowering time. The QTLs were subsequently analysed in different ecological conditions. A total of 21 QTLs, which were located on A02, C02, $\mathrm{C} 05$ and C08, were detected in three spring environments (Ledu, Xining and Huzhu). A total of 6 QTLs, which were distributed mainly on $\mathrm{A} 02, \mathrm{~A} 10$ and $\mathrm{C} 02$, were detected in the winter ecological conditions (Yuanmou) (Fig. 1). 
To verify the QTLs identified in multiple environments and integrate overlapping loci as consensus QTLs, BioMercator 2.1 software was used for a meta-analysis. Eighteen QTLs from the 27 QTLs detected in this study were integrated into 6 consensus QTLs as environmentally stable QTLs, and the remaining 9 QTLs were considered environment-specific QTLs (Table 2). The QTL cqFT-C02-3 was stable and detected in all 4 environments (one winter and three spring ecological conditions), explaining $3.83 \%$ to $21.61 \%$ of the phenotypic variance and -1.50 to -5.32 of the additive effects. A total of 4 QTLs (cqFT-A02-1, cqFT-A02-2, cqFT-C02-1 and cqFT-C02-2) were detected in the 3 spring environments; these QTLs explained 3.60$5.96 \%, 4.01-6.99 \%, 7.09-12.49 \%$ and $4.45-21.15 \%$ of the phenotypic variance, respectively. Nine QTLs for flowering time with no overlapping confidence intervals in the different environments were considered environment-specific QTLs, of which 4 were identified only in the spring ecological conditions and of which 5 were identified only in the winter ecological conditions.

The major QTLs explaining a relatively high proportion of the phenotypic variation are the focus of our research. There were 5 large-effect QTLs detected: sqFT-A02-3, sqFT-A02-4, sqFT-A10-1, cqFT-C02-2 and cqFT-C02-3 (Table 2, Fig. 2). The QTLs sqFT-A02-3, sqFT-A02-4 and sqFT-A10-1 explained $23.78 \%, 22.98 \%$ and $10.70 \%$ of the phenotypic variation, respectively. Three major QTLs were detected only in the winter ecological conditions, showing strong winter environmental specificity. The QTL cqFT-C02-2 explained $13.71 \%$ of the phenotypic variance and was detected in all 3 spring environments, showing strong spring environmental specificity. The major QTL cqFT-C02-3 was detected in all 4 environments and explained $11.02 \%$ of the phenotypic variance; this QTL was expressed both in the spring and winter ecological conditions. cqFT-C02-3 was considered an environmentally stable QTL. Regardless of whether these major QTLs were environmentally stable QTLs or environment-specific QTLs, they were all considered key QTLs for flowering time for subsequent fine mapping and map-based cloning analyses.

\section{Delimitation of the environment-specific major QTL sqFT-A02-4 target region}

In this study, several environment-specific QTLs with large-effect values were detected, and three of these major QTLs were detected only in winter ecological conditions, showing strong winter-environment specificity. The location of one of the major QTLs, sqFT-A02-4 (whose phenotypic variation exceeded 20\%), was delimited to a genetic region between markers Block9955 and Block10019 (Fig. 3a), corresponding to a candidate interval of approximately $216 \mathrm{~kb}$ between $6.302 \mathrm{Mb}$ and $6.518 \mathrm{Mb}$ on A02 of the $B$. napus genome (Fig. 3b). Therefore, the target gene may be located within the $216 \mathrm{~kb}$ range near the $6.3 \mathrm{Mb}$ position on $\mathrm{A} 02$. The sequence of the candidate region was analysed, in which a total of 27 predicted genes were included. Of these genes, the BnaA02g12130D/GSBRNA2T00090951001 gene was highly homologous to A. thaliana AT1G65480 (Fig. 3c). AT1G65480 is the FLOWERING LOCUS T(FT) gene in $A$. thaliana, which promotes flowering with $\angle E A F Y(L F Y)$ and is antagonistic to the TERMINAL FLOWER1 (TFL 1) gene; FT mutants exhibit late flowering. FT is a key gene involved in the photoperiod pathway and determines flowering time [27-29]. Therefore, we inferred that BnaA02g12130D (BnA02.FT) was the most important candidate gene for flowering time.

\section{Discussion}


Rapeseed (B. napus) originated along the Mediterranean coast approximately 7500 years ago. In China, the annual production of rapeseed oil reaches 3.5 million tons, accounting for $35 \%$ of China's edible vegetable oil. In China, rapeseed is distributed mainly in southern China (winter type) and on the QinghaiTibet Plateau (spring type). However, in recent years, the cultivation area of southern rapeseed has been continually declining, mainly because winter-type varieties have a long flowering time and a long maturity period that persists until mid- and early May of each year, which overlaps with the planting time of early rice and cotton crops grown in mid-to-late April. Eventually, most of the farmland is left idle and not used for agricultural production. The Qinghai-Tibet Plateau has an average elevation of more than 4000 metres and has a dry and cold climate, and only a short time is suitable for crop growth. Long flowering time can cause delayed maturity and the formation of abnormal or incompletely mature seeds, which subsequently lead to a decline in yield. The key to solving these problems is the breeding of rapeseed varieties with early-flowering as well as early-maturing phenotypes, which are urgently needed. In this study, we detected 27 QTLs in multiple environments, and the effects of these alleles may shorten the flowering time by 0.79 to 9.02 days. Among these QTLs, the major QTL cqFT-CO2-3 was identified in spring and winter ecological conditions as environmentally stable QTL that could shorten the flowering time of the late-flowering parent by 5.32 days in winter ecological conditions and shorten the flowing time by approximately 2 days in spring ecological conditions. The major QTL sqFT-A02-3 had an effect only in the winter ecological conditions, which could shorten the flowering time of the late-flowering parent by approximately 9 days. Study of the molecular mechanisms of these major QTLs would provide more theoretical references for breeding early-maturing rapeseed varieties.

Flowering time is an important agronomic trait. Constructing a mapping population is the key and foundation for QTL analysis. There are many kinds of mapping populations that can be used to construct linkage maps. According to their characteristics, there are two types of mapping populations: temporary and permanent ones. On the basis of the accuracy of QTL positioning, there are primary positioning genetic populations and fine positioning genetic populations. Specifically, they are divided into F2, backcross (BC), DH, and RIL as well as fine positioning populations of near-isogenic lines (NILs) and residual heterozygous lines (RHLs). RIL populations have been used to construct genetic linkage maps in rice [30]. RIL populations are constructed from F2s via single-grain transmission after multiple rounds of meiosis, and the degree of recombination is relatively high. Compared with $\mathrm{DH}$ populations, which are subject to severe partial segregation, RIL populations have higher accuracy and can be tested in a long-term and repeated manner. Likewise, we constructed a RIL population of 215 lines and planted them in both spring and winter ecological conditions. We ultimately used the genetic map constructed via this RIL population to identify multiple environmentally stable consensus QTLs.

QTL mapping of flowering time traits is one of the most effective ways to elucidate the genetic basis of flowering time and identify potential candidate genes underlying QTLs. There are currently many reports on QTL mapping of flowering genes in rapeseed, mainly in $B$. napus. Because of the differences in genome coverage, marker intensity, and specific markers applied to the maps, the genetic maps constructed by different populations significantly differ in terms of their QTL loci in different studies. For instance, Liu et al. [18] detected 3 consensus QTLs related to flowering time on the LG C2, but only 
cqDTFC02a was a major locus that explained $23.0 \%$ of the phenotypic variance. We submitted the flanking marker sequence of the cqDTFCO2a locus to the Brassica Database (BRAD) and compared it with the $B$. oleracea $\mathrm{CO} 2$ chromosome; the results showed that cqDTFCO2a was located at approximately $0.1 \mathrm{Mb}$ from the end of the $\mathrm{CO} 2$ chromosome in Brassica oleracea. Wei et al. [31] used a DH population of 261 lines and a genetic linkage map with simple sequence repeat (SSR) markers and detected QTLS ( $q F T 10-5$ and qFT11-7) for flowering time; these QTLs explained more than $20 \%$ of the phenotypic variance in the same environment for two consecutive years. We used the same method above for BLAST analysis and found that $q F T 10-5$ ( $q F T 11-7)$ was located at approximately $4.0 \mathrm{Mb}$ from the end of chromosome $\mathrm{CO} 2$ in B. oleracea. Moreover, we detected a major QTL, cqFT-C02-3, in all environments and conducted BLAST analysis on the $\mathrm{CO} 2$ chromosome of $B$. oleracea; our results showed that $c q F T-C 02-3$ is located at approximately $8 \mathrm{Mb}$ from the end of the $\mathrm{C} 02$ chromosome in $\mathrm{B}$. oleracea. Therefore, cqFT-C023 is different from the position of cqDTFCO2a and qFT10-5 (qFT11-7). With respect to the A02 chromosome, Li et al. [20] compared the QTLs for flowering time in different studies and found that the QTL Li-cqFT.A2-2 is a spring-specific QTL that colocalizes in the common genomic region with LongqFT2-6 [20, 32], and those authors found that this QTL is located within the A02 genomic region from 6.27-6.32 Mb and from 6.28-6.28 Mb, respectively. Our A02 chromosome analysis revealed two major specific QTLs in the winter ecological conditions: one of the major QTLs, sqFT-A02-4, was narrowed to a genomic region between $6.302 \mathrm{Mb}$ and $6.518 \mathrm{Mb}$ on chromosome $\mathrm{A} 02$ of $B$. napus. The genomic region of sqFT-A02-4 is close to that of both cqFT.A2-2 and Long-qFT2-6, so it is possible that sqFT-A02-4 is located in a common genomic region (Li-cqFT.A2-2 and Long-qFT2-6).

The whole-genome of $B$. napus has been sequenced. When the $B$. napus genomes was compared with the genomes of its progenitors $B$. rapa and $B$. oleracea, the $B$. napus genome was found to contain a remarkable evolutionary feature by which extensive DNA sequence exchange occurred between the $A$ and $C$ subgenomes [33]. Moreover, subgenomes $A$ and $C$ exhibit high synteny with each other, and these homologous genomic regions descend from a common ancestral genome and largely retain the same or similar genes, although some of them have undergone functional differentiation [34]. Studies have shown that the QTLs for flowering time are distributed on chromosomes A02 and $\mathrm{CO} 2$ in B. napus. Moreover, the QTLs display collinearity of the confidence intervals for chromosomes A02 and C02. For instance, Zhang et al. [35] performed a co-regional alignment of the confidence intervals of the detected flowering time QTLs on chromosomes A02 and C02, and multiple QTLs were localized in the A02-C02 syntenic regions and were subsequently referred to as syntenic QTLs. Therefore, the QTLs within coregions on chromosomes $\mathrm{A} 02$ and $\mathrm{C0} 2$ may indicate that these two syntenic regions are conserved and contain genes with the same or similar functions. For instance, Chen et al. [36] detected two major QTLs on $2 \mathrm{LGs}$ (A02 and C02). The two QTLs were subsequently delimited to the $86 \mathrm{~kb}$ and $201 \mathrm{~kb}$ regions via two separate near-isogenic populations. As a candidate gene, BnFLC was identified in both regions (A02 and C02) [36]. In the present study, ten QTLs were located on LG A02, and 13 QTLs were located on LG $\mathrm{C} 02$; the QTLs localized within the A02-C02 homologous regions will be analysed in subsequent studies.

Integrating QTLs with overlapping confidence intervals can help identify environmentally specific QTLs or multi-environmentally stable QTLs. In this study, 21 and 6 QTLs were detected in the spring ecological 
conditions and winter ecological conditions, respectively. In addition, QTLs were stably detected in the winter and spring ecological conditions. These environmentally stable QTLs could be beneficial for the breeding of new rapeseed varieties with broad adaptability. Environmentally stable QTLs have been reported in several studies; for instance, Liu et al. [18] discovered several flowering time QTLs in B. napus that were stably detected in five micro-environments. These QTLs were stable in different environments and are more suitable for fine mapping. Environment-specific QTLs have also been reported; for instance, Li et al. [20] investigated QTLs for flowering time in B. napus, and two major specific QTLs were detected in spring ecological conditions. These QTLs were expressed in a specific environment and were greatly affected by temperature and day length. In the present study, the major QTL cqFT-C02-3 was detected in all 4 environments and was considered environmentally stable. The major QTL cqFT-CO2-2 was detected in all 3 spring environments, showing strong spring environmental specificity. Therefore, whether these major QTLs were environmentally stable or environment specific, they were considered key QTLs for flowering time for future fine mapping and map-based cloning analyses.

Compared with reports on the QTL mapping for flowering time in B. napus, there are fewer reports concerning the cloning of genes responsible for flowering time. Currently, more than 180 genes related to flowering time have been detected in $A$. thaliana, and the majority of related research has focused on the $F L C, C O$, and $F T$ genes. The $A$ and $C$ genomes of $B$. napus are remarkably similar to those of $A$. thaliana. Based on the genome synteny between $A$. thaliana and $B$. napus, many $A$. thaliana homologous genes have been cloned in $B$. napus [37, 38]. For instance, Tadege et al. [39] cloned a homologue of $A$. thaliana $F L C$ in $B$. napus (BnFLC1-5); BnFLC1-5 was subsequently transformed into $A$. thaliana, and the flowering time of the transgenic plants was strongly delayed. Hou et al. [40] delimited the major QTL to an $80 \mathrm{~kb}$ interval and cloned a homologue of $A$. thaliana FLC in B. napus (BnFLC.A10). Similarly, Zheng et al. [41] cloned a homologue of $A$. thaliana $C O$ in $B$. napus (Bn1CON19), and the similarity between the Bn1CON19 gene sequence and the AtCO gene sequence reached $99.8 \%$. Robert et al. [42] identified three homologues of $A$. thaliana $C O$ in $B$. napus (BnCOA1, BnCOA9, BnCOB1 and BnCOB9), and Wang et al. [16] identified three $F T$ homologues in B. napus (BnFTA2, BnFTC6a and BnFTC6b) and 6 homologues of $A$. thaliana CO in B. napus. BnA2.FT, BnA7.FT.a, BnA7.FT.b, BnC2.FT, BnC6.FT and BnC6.FT.b. In addition, those authors reported that the variation in the BnFT allele might affect differences in flowering time between the winter and spring. $F T$ is considered a key gene that determines the flowering time in the photoperiod pathway, and $F T$ production leads to flowering-stimulating substances. When $A$. thaliana receives an appropriate photoperiod induction, the $C O$ gene initiates transcription and activates $F T$ expression; afterward, the $F T$ transcript (mRNA) moves from the leaves to the apical tissue, where the post-translational $F T$ protein and transcription factor protein FD interact to promote the expression of the downstream flowering gene $A P 1$, thereby promoting flowering $[14,15,43]$. In the present study, we selected BnA02.FT as a key candidate gene at a position close to $6.3 \mathrm{Mb}$ on chromosome $\mathrm{A} 02$ for further fine mapping and sequence analyses.

\section{Conclusions}


Five major novel QTLs for flowering time were discovered in spring Brassica napus: 1 environmentally stable QTL and 4 environment-specific QTLs. The major QTL sqFT-A02-4 was delimited to a candidate region of approximately $216 \mathrm{~kb}$, and $B n a A 02 g 12130 \mathrm{D}$ was determined to be a potential gene.

\section{Methods}

\section{Plant materials and methods}

A population consisting of 215 RILs derived from a cross between No. 3379 and No. 2839 was used for mapping. No. 3379 is an early-flowering spring-type B. napus, and No. 2839 is a late-flowering spring-type B. napus. No. 3379 usually blooms approximately 25-30 days sooner than does No. 2839. The RIL population was constructed as follows: No. 2839 was crossed with No. 3379 to obtain F1 plants. The F1 plants were self-pollinated to obtain F2 plants. Single-seed descendants were then propagated from the F2 generation to the F5 generation to obtain a RIL population consisting of 215 lines.

Field trials were conducted during the winter of 2017 at Yunnan and during the summer of 2018 at Qinghai, resulting in a total of 4 environments. The planting environment in winter 2017 included Yuanmou ( $898 \mathrm{~m}$ above sea level , 25 $42^{\circ} \mathrm{E}, 101^{\circ} 52^{\prime} \mathrm{N}$, Yunnan Province), and those in summer 2018 included Ledu (1978 $\mathrm{m}$ above sea level, $36.47^{\circ} \mathrm{E}, 102.43^{\circ} \mathrm{N}$, Qinghai province), Xining (2225 m above sea level, $36^{\circ} 34^{\prime} \mathrm{E}, 101^{\circ} 49^{\prime} \mathrm{N}$, Qinghai province) and Huzhu ( $2557 \mathrm{~m}$ above sea level, 36 $49 \mathrm{E}, 101^{\circ} 57 \mathrm{~N}$, Qinghai province). The RIL population, F1 population and the parents (No. 3379 and No. 2839) were evaluated as part of a randomized complete block design with three replicates in each environment. The experimental plot comprised two rows, each $1.5 \mathrm{~m}$ in length and $0.3 \mathrm{~m}$ in width. There were ten plants per row, and the spacing was $866 \mathrm{~m}^{2}$. The fields were managed in accordance with local standard agricultural practices.

\section{Phenotypic evaluations}

The flowering time was calculated by subtracting the date of seedling emergence from the flowering date. The date of cotyledon flattening was recorded as the seedling emergence date of each plant. The seedling emergence in a single plot was considered the date when $75 \%$ of the plants in the plot reached seedling emergence. The date of the first flower per plant was recorded as the flowering date of each individual, and the flowering time in a single plot was considered the date when $25 \%$ of the plants in the plot had flowered.

\section{Statistical analysis of phenotypic data}

Various modules of the Statistical Analysis System (SPSS 19.0) software package were used to analyse the phenotypic data. Broad-sense heritability $\left(h^{2}\right)$ was calculated as $\sigma^{2}{ }_{g} /\left(\sigma^{2}{ }_{g}+\sigma_{g e}^{2} / n+\sigma^{2}{ }_{e} / n r\right) \times$ $100 \%$, where $\sigma_{g}^{2}$ is the genetic variance, $\sigma^{2}$ is the error variance, $\sigma^{2}$ ge is the interaction variance between genotypes and environments, $\mathrm{n}$ is the number of environments, and $\mathrm{r}$ is the number of replications in each environment. The estimates of $\sigma^{2}{ }_{g}, \sigma^{2}$ ge and $\sigma^{2}$ were obtained from a two-way ANOVA using the general linear model (GLM) procedure in SAS 8.1. In the variance analysis model, the variances of the genotypes, 
environments and interactions between the genotypes and environments were considered random effects. Correlations were calculated for the flowering time between the four environments by SAS 8.1 software.

\section{Library construction and high-throughput sequencing}

The genomic DNA of the 215 RILs and their parents was extracted from fresh leaves $(100 \mathrm{mg})$ using the cetyl-trimethylammonium bromide (CTAB) method. The quality of the DNA samples was measured, and the fragments was randomly interrupted by sonication to a size of 200-500 bp. The sequencing libraries were constructed by terminal repair followed by the addition of a $3^{\prime} A$ and a sequencing adapter. The resulting fragments were further purified and amplified by PCR. After quality inspection, the constructed library was subjected to paired-end sequencing by an Illumina HiSeq 2500 system (Illumina, San Diego, CA, USA) following the manufacturer's instructions. Re-sequencing was performed by the Biomarker Technologies Company (Beijing, China).

\section{Identification of SNPs and genotyping}

The original reads were processed in accordance with the following quality control steps: 1) the sequences of the adapter were removed; 2 ) reads with $10 \%$ or more unidentified nucleotides were removed; and 3) reads having more than $50 \%$ bases with a phred quality lower than $10 \%$ were removed. Burrows Wheeler Aligner (BWA) was subsequently applied to align the clean reads of each material to the B. napus reference genome (http://www.genoscope.cns.fr/brassicanapus/).

Potential PCR duplications were eliminated using the MARK duplicate tool of Picard [44]. The GATK software toolkit 3.8 was then used to detect potential SNPs among the lines [45]; this process included base recalibration, variation calling, and strict filtering of SNPs to obtain a final SNP cluster. SnpEff software was used for variation (SNP and small InDel) annotation and for the prediction of variation effects [46].

To ensure the quality of the genetic map, markers were screened in accordance with the following three criteria: 1) only the aa'bb genotype was kept based on the SNPs of two parents, 2) the SNPs with a depth $\geq 1$ in each parent were selected, and 3) the SNPs that were not located on chromosomes were removed.

\section{Linkage map construction}

Filling and correction of SNPs were carried out using a sliding window analysis, with 15 SNPs per window and 1 SNP per increment. The genotype of the window was identified as aa when there were 11 or more SNPs with the aa genotype in a sliding window. When the genotype of 11 or more SNPs in the sliding window was bb, the genotype of this window was classified as bb. Except for the above 2 cases, the genotype of a window was otherwise identified as ab.

Bin partitioning was subsequently performed according to the sub-reorganization. Each sample was arranged according to its physical position on the chromosome. When there was a genotype transition in 
any sample, a recombination breakpoint appeared. The SNP between the recombination breakpoints was included in the bin, and it was supposed that no recombination events occurred in the bin. The bins were subsequently used as mapping markers for genetic map construction [47].

According to the distribution of markers, bins with a length less than $10 \mathrm{~kb}$ were removed from LGs A09, $\mathrm{CO3}, \mathrm{CO4}$, and C07, and bins with a length less than $5 \mathrm{~kb}$ were removed from the other LGs. The markers showing severe segregation distortion were filtered by specific parameters. The filtration parameter for $A 01, C 03, C 04$ and CO7 is 0.001; the parameter for A03, A04, A05, A10, C01, C05, C06 and C09 is 0.01; the parameter for $\mathrm{A} 06, \mathrm{~A} 07, \mathrm{~A} 08$ and $\mathrm{C} 08$ is 0.00001 ; and the markers on the remaining chromosomes were not filtered.

All bin markers of the linkage map corresponded to the physical sequences of the reference genome of $B$. napus. The collinearity between the genetic location and physical location was determined by mapping the relationship between the genetic marker positions (cM) and the physical positions ( $\mathrm{Mb})$.

\section{QTL identification and analysis}

QTL mapping was performed by the composite interval mapping (CIM) method in Windows QTL Cartographer 2.5 software [48]. The LOD thresholds for determining significant loci were estimated from 1000 permutations [49]. The LOD threshold was set to 2.5 to detect significant QTLs for flowering time. The confidence interval for each QTL was defined by a LOD change from the peak position - when multiple peaks are close to each other and within a half LOD distance. The identified QTLs were named according to the different environments and chromosome numbers [50,51]. The consensus QTLs were integrated by a meta-analysis by BioMercator 2.1 software [52]. If a QTL was identified only in one environment, then that QTL was considered an environment-specific QTL. If a QTL was identified simultaneously in the spring and winter ecological conditions or in multiple spring environments, those QTLs were thus located at a designated position on the same chromosome and were treated as environmentally stable consensus QTLs. A QTL explaining more than $10 \%$ of the phenotypic variance was considered a major QTL.

\section{Select candidate gene}

The candidate interval of the major QTLs with overlapping physical regions in specific environments were selected for subsequent analysis. Based on the positions of the flanking bin markers, all of the genes within the confidence interval were identified as candidates, and the sequences of the candidate regions were subsequently submitted to the $B$. napus genome resource (http://www.genoscope.cns.fr/brassicanapus/), the BRAD (http://www.brassicadb.org/brad/) and the Arabidopsis Information Resource (TAIR) (https://www.arabidopsis.org/) databases for BLAST analysis. The candidate genes of the target loci regions were predicted by combining the gene annotation of the BnaA02g12130D reference genome and the homologous gene annotation related to the flowering time of A. thaliana. 


\section{Abbreviations}

QTL: quantitative trait locus; SNPs: single-nucleotide polymorphisms; LGs: linkage groups; GA: gibberellic acid; WGRS: whole-genome re-sequencing; Indels: insertions/deletions; SV: structure variation; CNV: copy number variation; ANOVAs: analyses of variance; G: genotype; E: environment; $h^{2}$ : The broad-sense heritability; RIL: recombinant inbred line; DH: double-haploid; BC: back-cross; NILs: near-isogenic lines; RHLs: residual heterozygous lines; BRAD: Brassica Database; SSR: simple sequence repeat; GLM: general linear model; BWA: Burrows Wheeler Aligner; TAIR: the Arabidopsis Information Resource.

\section{Declarations}

\section{Ethics approval and consent to participate}

Not applicable.

\section{Consent for publication}

Not applicable.

\section{Availability of data and materials}

All the data generated and analysed during this study are included within the article and its additional files.

\section{Competing interests}

The authors declare that they have no competing interests.

\section{Funding}

This research was financially supported by the National Key Research and Development Plan of China (2016YFD0101300), the Breeding of New Transgenic Rapeseed Varieties in Spring Rapeseed Area (2018zx08020001-009), the National System of Technology of the Rapeseed Industry (CARS-12), the Key Laboratory of Spring Rape Genetic Improvement of Qinghai Province (2017-ZJ-Y09) and the Qinghai Natural Science Foundation Project (2017-ZJ-941Q).

\section{Authors' contributions}

LX and DD conceived the research and designed the experiments. $X L$ performed most of the experiments and was a major contributor in writing the manuscript, QM and LL participated in part of the field phenotypic investigation, ZZ and GT participated in field management, HL participated in the data analysis. All authors read and approved the final manuscript.

\section{Acknowledgements}


We are grateful to Prof. Zhong Fu and Mr. Jianshe Qin for providing help with field management. We are also thank Handong Wang (Northwest Institute of Plateau Biology, Chinese Academy of Sciences) for assistance with data analysis.

\section{Authors' information}

${ }^{1}$ Academy of Agricultural and Forestry Sciences of Qinghai University, State Key Laboratory of Plateau Ecology and Agriculture of Qinghai University, Key Laboratory of Qinghai Province for Spring Rapeseed Genetic Improvement, The Qinghai Research Branch of the National Rapeseed Genetic Improvement Center, Spring Rape Scientific Observation Experimental Station of Ministry of Agriculture and Rural Areas, Qinghai Spring Rape Engineering Research Center, Xining 810016, China.

\section{References}

1. Shyam P, Wu XM, Bhat SR. History, evolution, and domestication of brassica crops. Plant Breed Rev. 2011;35:19-84.

2. Wang N, Qian W, Suppanz I, Wei L, Mao B, Long Y, et al. Flowering time variation in oilseed rape (Brassica napus L.) is associated with allelic variation in the FRIGIDA homologue BnaA. FRI. J Exp Bot, 2011; 62(15):5641-5658.

3. Guan CY, Chen SY, Wu ML. Research evolution on breeding and mechanical cultivation of earlymature winter rapeseed in double-crop rice area in southern China. Eng Sci. 2010;12:4-10.

4. Du DZ, Nie P, Xu L, Luo YX, Yao YM, Zhou HW, et al. Rapeseed heterosis of different ecotypes in Qinghai province. Chin J Oil Crop Sci. 2010;32:180-6.

5. Zhao JY, Becker HC, Ding HD, Zhang YF, Zhang DQ, Ecke W. QTL of three agronomically important traits and their interactions with environment in a European $\mathrm{x}$ Chinese rapeseed population. Yi Chuan Xue Bao. 2005;32:969-78.

6. Andres F, Coupland G. The genetic basis of flowering responses to seasonal cues. Nat Rev Genet. 2012;13:627-39.

7. Baurle I, Dean C. The timing of developmental transitions in plants. Cell. 2006;125:655-64.

8. Amasino RM, Michaels SD. The timing of flowering. Plant Physiol. 2010;154:516-20.

9. Srikanth A, Schmid M. Regulation of flowering time: all roads lead to Rome. Cell Mol Life Sci. 2011;68:2013-37.

10. Wang JW. Regulation of flowering time by the miR156-mediated age pathway. J Exp Bot. 2014;65:4723-30.

11. Mouradov A, Cremer F, Coupland G. Control of flowering time: interacting pathways as a basis for diversity. Plant Cell. 2002;14 Suppl:S111-30.

12. Fornara F, de Montaigu A, Coupland G. SnapShot: control of flowering in Arabidopsis. Cell. 2010;141:550, 550.e1-2. 
13. Abe M, Kobayashi Y, Yamamoto S, Daimon Y, Yamaguchi A, lkeda Y, et al. FD, a bZIP protein mediating signals from the floral pathway integrator FT at the shoot apex. Science. 2005;309:1052-6.

14. Wigge PA, Kim MC, Jaeger KE, Busch W, Schmid M, Lohmann JU, et al. Integration of spatial and temporal information during floral induction in Arabidopsis. Science. 2005;309:1056-9.

15. Wigge PA. FT, a mobile developmental signal in plants. Curr Biol. 2011;21:R374-8.

16. Wang J, Long Y, Wu B, Liu J, Jiang C, Shi L, et al. The evolution of Brassica napus FLOWERING LOCUST paralogues in the context of inverted chromosomal duplication blocks. BMC Evol Biol. 2009;9:271.

17. Wang XD, Chen L, Wang A, Wang H, Tian JH, Zhao XP, et al. Quantitative trait loci analysis and genome-wide comparison for silique related traits in Brassica napus. BMC Plant Biol. 2016;16:71.

18. Liu H, Du D, Guo S, Xiao L, Zhao Z, Zhao Z, et al. QTL analysis and the development of closely linked markers for days to flowering in spring oilseed rape (Brassica napus L.). Mol Breed. 2016;36:52.

19. Xu L, Hu K, Zhang Z, Guan C, Chen S, Hua W, et al. Genome-wide association study reveals the genetic architecture of flowering time in rapeseed (Brassica napus L.). DNA Res. 2016;23:43-52.

20. Li B, Zhao W, Li D, Chao H, Zhao X, Ta N, et al. Genetic dissection of the mechanism of flowering time based on an environmentally stable and specific QTL in Brassica napus. Plant Sci. 2018;277:296310 .

21. Schmutz J, McClean PE, Mamidi S, Wu GA, Cannon SB, Grimwood J, et al. A reference genome for common bean and genome-wide analysis of dual domestications. Nat Genet. 2014;46:707-13.

22. Wang L, Yu S, Tong C, Zhao Y, Liu Y, Song C, et al. Genome sequencing of the high oil crop sesame provides insight into oil biosynthesis. Genome Biol. 2014;15:R39.

23. Davey JW, Hohenlohe PA, Etter PD, Boone JQ, Catchen JM, Blaxter ML. Genome-wide genetic marker discovery and genotyping using next-generation sequencing. Nat Rev Genet. 2011;12:499-510.

24. Takagi H, Abe A, Yoshida K, Kosugi S, Natsume S, Mitsuoka C, et al. QTL-seq: rapid mapping of quantitative trait loci in rice by whole genome resequencing of DNA from two bulked populations. Plant J. 2013;74:174-83.

25. Xu Q, Shi Y, Yu T, Xu X, Yan Y, Qi X, et al. Whole-Genome resequencing of a cucumber chromosome segment substitution line and its recurrent parent to identify candidate genes governing powdery mildew resistance. PLoS One. 2016;11:e0164469.

26. Liu HL. Origin and evolution of rapeseeds. Acta Agron Sin. 1984;10:9-18.

27. Kobayashi Y, Kaya H, Goto K, Iwabuchi M, Araki T. A pair of related genes with antagonistic roles in mediating flowering signals. Science. 1999;286:1960-2.

28. Ahn JH, Miller D, Winter VJ, Banfield MJ, Lee JH, Yoo SY, et al. A divergent external loop confers antagonistic activity on floral regulators FT and TFL1. EMBO J. 2006;25:605-14.

29. Bohlenius H, Huang T, Charbonnel-Campaa L, Brunner AM, Jansson S, Strauss SH, et al. CO/FT regulatory module controls timing of flowering and seasonal growth cessation in trees. Science. 2006;312:1040-3. 
30. Zhuang JY, Fan YY, Rao ZM, Wu JL, Xia YW, Zheng KL. Analysis on additive effects and additive-byadditive epistatic effects of QTLs for yield traits in a recombinant inbred line population of rice. Theor Appl Genet. 2002;105:1137-45.

31. Wei D, Mei J, Fu Y, Disi JO, Li J, Qian W. Quantitative trait loci analyses for resistance to Sclerotinia sclerotiorum and flowering time in Brassica napus. Mol Breed. 2014;34:1797-804.

32. Long Y, Shi J, Qiu D, Li R, Zhang C, Wang J, et al. Flowering time quantitative trait Loci analysis of oilseed brassica in multiple environments and genomewide alignment with Arabidopsis. Genetics. 2007;177:2433-44.

33. Chalhoub B, Denoeud F, Liu S, Parkin IA, Tang H, Wang X, et al. Plant genetics. Early allopolyploid evolution in the post-Neolithic Brassica napus oilseed genome. Science. 2014;345:950-3.

34. Panjabi P, Jagannath A, Bisht NC, Padmaja KL, Sharma S, Gupta V, et al. Comparative mapping of Brassica juncea and Arabidopsis thaliana using Intron Polymorphism (IP) markers: homoeologous relationships, diversification and evolution of the A, B and C Brassica genomes. BMC Genomics. 2008;9:113.

35. Zhang F, Huang J, Tang M, Cheng X, Liu Y, Tong C, et al. Syntenic quantitative trait loci and genomic divergence for Sclerotinia resistance and flowering time in Brassica napus. J Integr Plant Biol. 2019;61:75-88.

36. Chen L, Dong F, Cai J, Xin Q, Fang C, Liu L, et al. A 2.833-kb insertion in BnFLC.A2 and its homeologous exchange with BnFLC.C2 during breeding selection generated early-flowering rapeseed. Mol Plant. 2018;11:222-5.

37. Yang YW, Lai KN, Tai PY, Li WH. Rates of nucleotide substitution in angiosperm mitochondrial DNA sequences and dates of divergence between Brassica and other angiosperm lineages. J Mol Evol. 1999;48:597-604.

38. Xiao L, Li X, Liu F, Zhao Z, Xu L, Chen C, et al. Mutations in the CDS and promoter of BjuA07.CLV1 cause a multilocular trait in Brassica juncea. Sci Rep. 2018;8:5339.

39. Tadege M, Sheldon CC, Helliwell CA, Stoutjesdijk P, Dennis ES, Peacock WJ. Control of flowering time by FLC orthologues in Brassica napus. Plant J. 2001;28:545-53.

40. Hou J, Long Y, Raman H, Zou X, Wang J, Dai S, et al. A Tourist-like MITE insertion in the upstream region of the BnFLC.A10 gene is associated with vernalization requirement in rapeseed (Brassica napus L.). BMC Plant Biol. 2012;12:238.

41. Zheng BC, Zhang JF, Li HJ, Chai L, Cui C, Jiang J, et al. Analysis of quantitative expression of the flowering-regulating transcription factor CONSTANS gene in Brassica napus L. Scientia Agricultura Sinica. 2013;46:2592-8.

42. Robert LS, Robson F, Sharpe A, Lydiate D, Coupland G. Conserved structure and function of the Arabidopsis flowering time gene CONSTANS in Brassica napus. Plant Mol Biol. 1998;37:763-72.

43. Bernier G. The Control of floral evocation and morphogenesis. Annu Rev Plant Physiol Plant Mol Biol. 1988;39:175-219.

44. Picard. https://sourceforge.net/projects/picard/. Accessed 7 Aug 2015. 
45. DePristo MA, Banks E, Poplin R, Garimella KV, Maguire JR, Hartl C, et al. A framework for variation discovery and genotyping using next-generation DNA sequencing data. Nat Genet. 2011;43:491-8.

46. Cingolani P, Platts A, Wang L, Coon M, Nguyen T, Wang L, et al. A program for annotating and predicting the effects of single nucleotide polymorphisms, SnpEff: SNPs in the genome of Drosophila melanogaster strain w1118; iso-2; iso-3. Fly (Austin). 2012;6:80-92.

47. Huang X, Feng Q, Qian Q, Zhao Q, Wang L, Wang A, et al. High-throughput genotyping by wholegenome resequencing. Genome Res. 2009;19:1068-76.

48. Wang S, Basten CJ, Zeng ZB. Windows QTL cartographer version 2.5. Statistical genetics. North Carolina State University, Raleigh, NC. 2005.

49. Churchill GA, Doerge RW. Empirical threshold values for quantitative trait mapping. Genetics. 1994;138:963-71.

50. Chen G, Geng J, Rahman M, Liu X, Tu J, Fu T, et al. Identification of QTL for oil content, seed yield, and flowering time in oilseed rape (Brassica napus). Euphytica. 2010;175:161-74.

51. Burns MJ, Barnes SR, Bowman JG, Clarke MH, Werner CP, Kearsey MJ. QTL analysis of an intervarietal set of substitution lines in Brassica napus: (i) seed oil content and fatty acid composition. Heredity (Edinb). 2003;90:39-48.

52. Goffinet B, Gerber S. Quantitative trait loci: a meta-analysis. Genetics. 2000;155:463-73.

\section{Tables}

Table 1 Descriptive statistics of flowering time in the parents and in the F1 and RIL populations.

\begin{tabular}{|c|c|c|c|c|c|c|c|}
\hline \multirow[t]{2}{*}{ Environment $^{\mathrm{a}}$} & \multicolumn{2}{|c|}{ Parents } & \multirow[t]{2}{*}{ F1 } & \multicolumn{4}{|c|}{ RILs } \\
\hline & No. 3379 & No. 2839 & & Range & Mean $\pm \mathrm{SD}^{\mathrm{b}}$ & Skewness & Kurtosis \\
\hline $18 \mathrm{HZ}$ & $36.33 \pm 0.85$ & $61.00 \pm 1.00^{* *}$ & $45.67 \pm 0.58$ & $40.00-59.67$ & $49.47 \pm 4.28$ & 0.16 & -0.31 \\
\hline $18 \mathrm{XN}$ & $30.33 \pm 1.15$ & $61.33 \pm 0.58^{* *}$ & $40.67 \pm 1.15$ & $34.67-59.67$ & $45.23 \pm 4.96$ & 0.55 & -0.03 \\
\hline $18 \mathrm{LD}$ & $30.00 \pm 1.00$ & $60.33 \pm 1.53^{* *}$ & $41.50 \pm 2.12$ & $35.00-60.00$ & $47.09 \pm 5.56$ & 0.34 & -0.61 \\
\hline $17 \mathrm{YM}$ & $19.00 \pm 1.00$ & $112.33 \pm 4.73^{* *}$ & $34.33 \pm 1.53$ & $27.67-108.67$ & $51.76 \pm 17.00$ & 0.61 & -0.35 \\
\hline
\end{tabular}

Note: ${ }^{* *}$ represents the 0.01 level of significance between both parents in the same environment based on a t test; a represents the environment; b means the standard deviation (SD).

Table 2 Information about consensus QTLs, specific QTLs and identified QTLs for flowering time within the RIL population in 2017 and 2018. 


\begin{tabular}{|c|c|c|c|c|c|c|c|c|c|c|c|}
\hline $\begin{array}{l}\text { Consensus/Specific } \\
\text { QTLs } \\
\end{array}$ & $\begin{array}{l}\text { Peak } \\
(\mathrm{cM}) \\
\end{array}$ & CI (cM) & $\begin{array}{l}\text { Identified } \\
\text { QTLs } \\
\end{array}$ & LG & LOD & $\begin{array}{l}\mathrm{R}^{2} \\
(\%) \\
\end{array}$ & ADD & $\begin{array}{l}\text { Peak } \\
(\mathrm{cM}) \\
\end{array}$ & CI (cM) & Environment & $\begin{array}{l}\text { QTL } \\
\text { type }\end{array}$ \\
\hline \multirow[t]{5}{*}{ CqFT-A02-1 } & 87.79 & $86.46-89.12$ & $\begin{array}{l}q F T A 02- \\
18 H Z 1\end{array}$ & A02 & 3.08 & 3.60 & -0.85 & 88.31 & $86.5-91.2$ & Spring & Minor \\
\hline & & & & A02 & 4.27 & 5.58 & -1.23 & 88.31 & 86.8-93.3 & & \\
\hline & & & qFTA02- & & & & & & & & \\
\hline & & & $18 X N 1$ & A02 & 4.41 & 5.96 & -1.41 & 87.31 & 86.4-90.1 & & \\
\hline & & & $\begin{array}{l}\text { qFTA02- } \\
18 L D 1\end{array}$ & & & & & & & & \\
\hline \multirow[t]{7}{*}{ cqFT-A02-2 } & 97.43 & $\begin{array}{l}94.69- \\
100.16\end{array}$ & $\begin{array}{l}\text { qFTA02- } \\
18 H Z 2\end{array}$ & A02 & 3.45 & 4.01 & -0.94 & 97.71 & $91.2-99.4$ & Spring & Minor \\
\hline & & & & A02 & 4.31 & 5.63 & -1.29 & 97.71 & $94.7-$ & & \\
\hline & & & qFTAO2- & & & & & & 106.7 & & \\
\hline & & & $18 X N 2$ & A02 & 5.21 & 6.99 & -1.60 & 96.91 & & & \\
\hline & & & & & & & & & 90.1-99.4 & & \\
\hline & & & qFTA02- & & & & & & & & \\
\hline & & & $18 L D 2$ & & & & & & & & \\
\hline \multirow[t]{5}{*}{$C q F T-C 02-1$} & 35.63 & $35.15-36.11$ & $\begin{array}{l}\text { qFTCO2- } \\
18 H Z 1\end{array}$ & $\mathrm{C} 02$ & 5.18 & 7.35 & -1.54 & 36.11 & $35.4-36.8$ & Spring & Minor \\
\hline & & & & $\mathrm{C} 02$ & 4.95 & 7.09 & -1.82 & 35.21 & $34.4-36.2$ & & \\
\hline & & & qFTC02- & & & & & & & & \\
\hline & & & $18 X N 1$ & $\mathrm{C} 02$ & 7.75 & 12.49 & -2.49 & 35.21 & $34.9-35.4$ & & \\
\hline & & & $\begin{array}{l}q F T C 02- \\
18 L D 1\end{array}$ & & & & & & & & \\
\hline \multirow[t]{6}{*}{ CqFT-CO2-2 } & 42.08 & $41.34-42.82$ & $\begin{array}{l}\text { qFTCO2- } \\
18 H Z 2\end{array}$ & $\mathrm{C} 02$ & 11.76 & 15.54 & -2.13 & 44.41 & $43.1-47.8$ & Spring & Major \\
\hline & & & & $\mathrm{C} 02$ & 3.43 & 4.45 & -1.59 & 42.81 & $40.8-48.1$ & & \\
\hline & & & qFTC02- & & & & & & & & \\
\hline & & & $18 X N 2$ & $\mathrm{C} 02$ & 14.02 & 21.15 & -2.82 & 41.51 & $40.8-42.5$ & & \\
\hline & & & qFTCO2- & & & & & & & & \\
\hline & & & $18 L D 2$ & & & & & & & & \\
\hline \multirow[t]{7}{*}{ CqFT-CO2-3 } & 53.32 & $52.65-53.99$ & $\begin{array}{l}\text { qFTCO2- } \\
18 \mathrm{HZ3}\end{array}$ & $\mathrm{C} 02$ & 9.56 & 12.93 & -1.87 & 54.11 & $52.7-54.3$ & $\begin{array}{l}\text { Spring, } \\
\text { Winter }\end{array}$ & Major \\
\hline & & & & $\mathrm{C} 02$ & 2.93 & 3.83 & -1.50 & 50.51 & 48.1-53.0 & & \\
\hline & & & qFTC02- & & & & & & & & \\
\hline & & & $18 X N 3$ & $\mathrm{C} 02$ & 14.37 & 21.61 & -2.78 & 50.51 & $48.3-52.8$ & & \\
\hline & & & $\begin{array}{l}\text { qFTCO2- } \\
18 L D 3\end{array}$ & $\mathrm{C} 02$ & 4.80 & 5.72 & -5.32 & 52.71 & $52.0-55.5$ & & \\
\hline & & & qFTCO2- & & & & & & & & \\
\hline & & & $17 Y M 1$ & & & & & & & & \\
\hline \multirow[t]{3}{*}{ CqFT-C05-1 } & 37.91 & $35.47-40.36$ & $\begin{array}{l}\text { qFTCO5- } \\
18 H Z 1\end{array}$ & $\mathrm{C} 05$ & 2.83 & 3.47 & -0.79 & 39.31 & $31.2-39.6$ & Spring & Minor \\
\hline & & & & $\mathrm{C} 05$ & 2.91 & 3.83 & -1.11 & 37.21 & $33.6-39.6$ & & \\
\hline & & & $\begin{array}{l}q F T C 05- \\
18 L D 1\end{array}$ & & & & & & & & \\
\hline$s q F T-A 02-1$ & 108.11 & 107.9-110.4 & $\begin{array}{l}\text { qFTA02- } \\
18 X N 3\end{array}$ & A02 & 2.76 & 3.67 & -1.05 & 108.11 & $\begin{array}{l}107.9- \\
110.4\end{array}$ & Spring & Minor \\
\hline$s q F T-A 02-2$ & 104.81 & 102.5-107.9 & $\begin{array}{l}\text { qFTA02- } \\
18 L D 3\end{array}$ & A02 & 3.57 & 4.87 & -1.38 & 104.81 & $\begin{array}{l}102.5- \\
107.9\end{array}$ & Spring & Minor \\
\hline$s q F T-A 02-3$ & 30.51 & 29.3-31.4 & $\begin{array}{l}\text { qFTA02- } \\
17 Y M 1\end{array}$ & A02 & 16.20 & 23.78 & -9.02 & 30.51 & 29.3-31.4 & Winter & Major \\
\hline SqFT-A02-4 & 37.61 & $36.6-39.8$ & $\begin{array}{l}\text { qFTAO2- } \\
17 Y M 2\end{array}$ & $\mathrm{~A} 02$ & 15.81 & 22.98 & -8.34 & 37.61 & $36.6-39.8$ & Winter & Major \\
\hline$s q F T-A 02-5$ & 24.11 & $20.1-24.5$ & $\begin{array}{l}\text { qFTA02- } \\
17 Y M 3\end{array}$ & A02 & 2.80 & 3.25 & -3.94 & 24.11 & 20.1-24.5 & Winter & Minor \\
\hline
\end{tabular}




\begin{tabular}{|c|c|c|c|c|c|c|c|c|c|c|c|}
\hline$s q F T-A 10-1$ & 52.91 & 48.7-53.5 & $\begin{array}{l}q F T A 10- \\
17 Y M 1\end{array}$ & A10 & 8.67 & 10.70 & -5.89 & 52.91 & 48.7-53.5 & Winter & Major \\
\hline$s q F T-C 02-1$ & 26.31 & $20.5-28.0$ & $\begin{array}{l}q F T C 02- \\
18 X N 4\end{array}$ & $\mathrm{C} 02$ & 2.64 & 3.39 & -1.33 & 26.31 & $20.5-28.0$ & Spring & Minor \\
\hline$s q F T-C 02-2$ & 71.51 & 68.3-74.8 & $\begin{array}{l}q F T C 02- \\
17 Y M 2\end{array}$ & $\mathrm{C} 02$ & 4.65 & 5.55 & -5.23 & 71.51 & 68.3-74.8 & Winter & Minor \\
\hline$s q F T-C 08-1$ & 14.41 & $10.6-18.2$ & $\begin{array}{l}q F T C 08- \\
18 L D 1\end{array}$ & $\mathrm{C} 08$ & 2.86 & 3.76 & -1.09 & 14.41 & $10.6-18.2$ & Spring & Minor \\
\hline
\end{tabular}

Note: spring ecological conditions: HZ (Huzhu), XN (Xining) and LD (Ledu); winter ecological conditions: YM (Yuanmou); planting year: 2017 and 2018; CI: confidence interval of the QTL; LG: specific linkage group; $\mathrm{R}^{2}$ : phenotypic variation explained by the QTL; ADD: additive effect of the QTL; Environment: ecological conditions; LOD: logarithm of odds score at the QTL peak; QTL type: whether the QTL is major or minor.

\section{Figures}




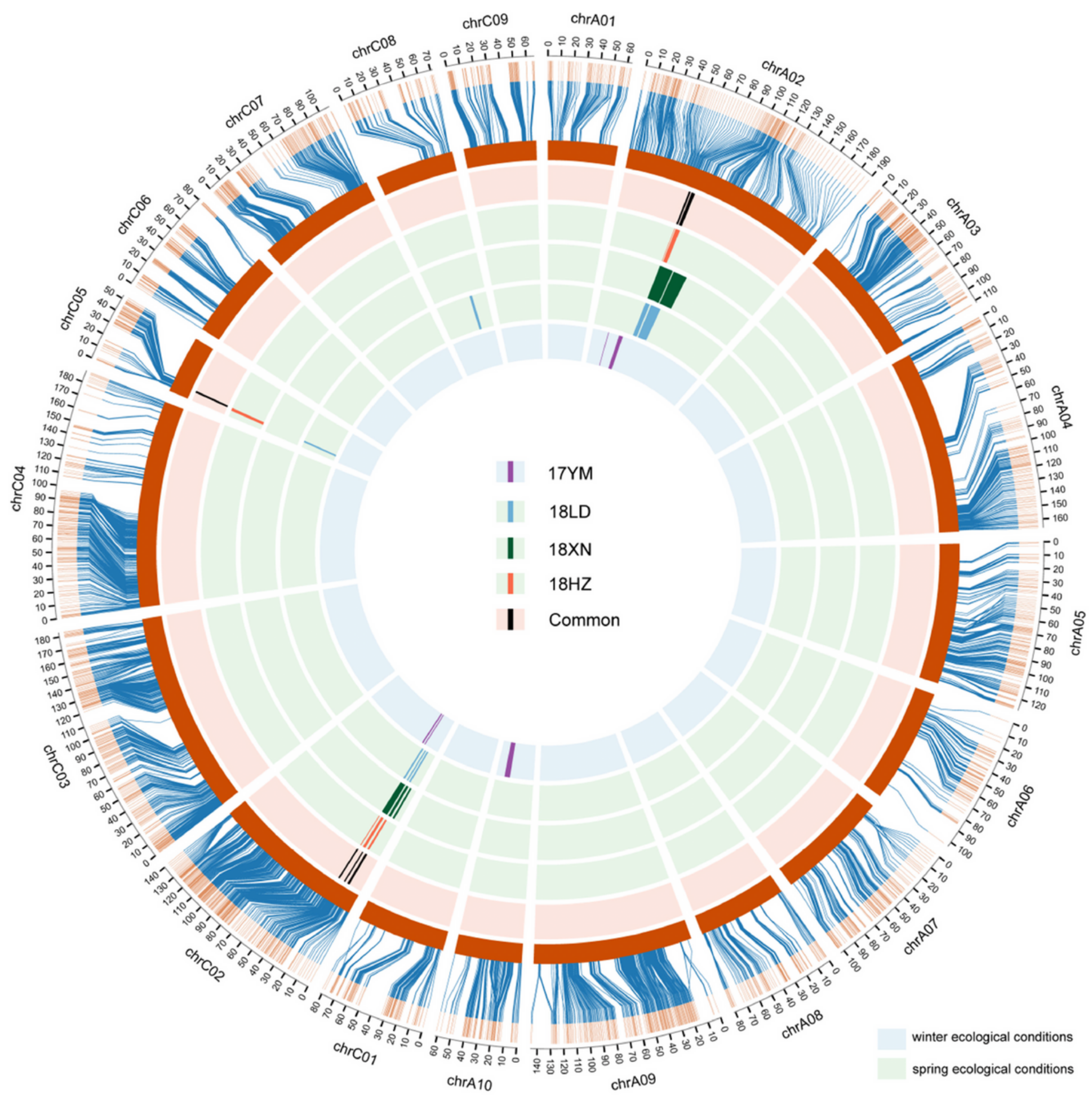

\section{Figure 1}

Distribution of bin markers on the linkage map and the QTL information of flowering time in the four environments. The figure shows the physical position of the bin markers on each LG (from the outermost circle to the second outer circle). The 27 flowering time QTLs identified in three spring ecological conditions (light-green circle) and in one winter ecological condition (light-blue circle) were distributed among five LGs. The different environments and years are indicated by years + location (from the fourth outer circle to the seventh circle: $18 \mathrm{HZ}, 18 \mathrm{XN}, 18 \mathrm{LD}$ and 17YM). The 6 consensus QTLs are located on the 
third pink circle. Specific QTLs were identified by single-environment analysis in the four environments. The ranges of the blocks represent the confidence interval of each QTL (colour figure available online)
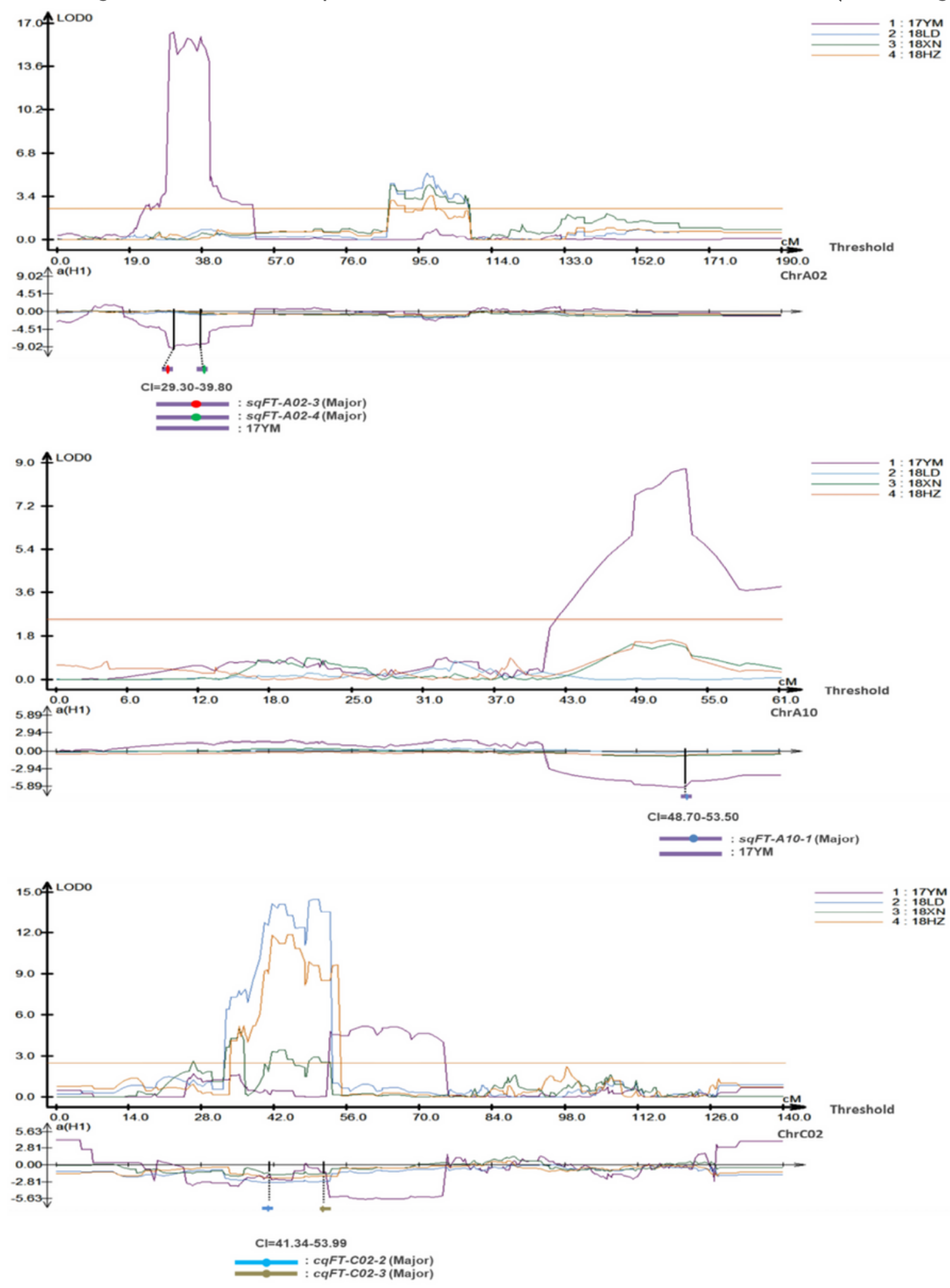

Figure 2

QTLs scanning results for flowering time QTLs on A02, $\mathrm{C02}$ and A10. The various colour curves above represent the QTLs detected in different environments, and the various colour curves below represent the additive effect values of the QTLs in the different environments. The major and minor consensus QTLs 
are shown under the confidence interval, and the major specific QTLs are shown under the confidence interval in a specific environment.

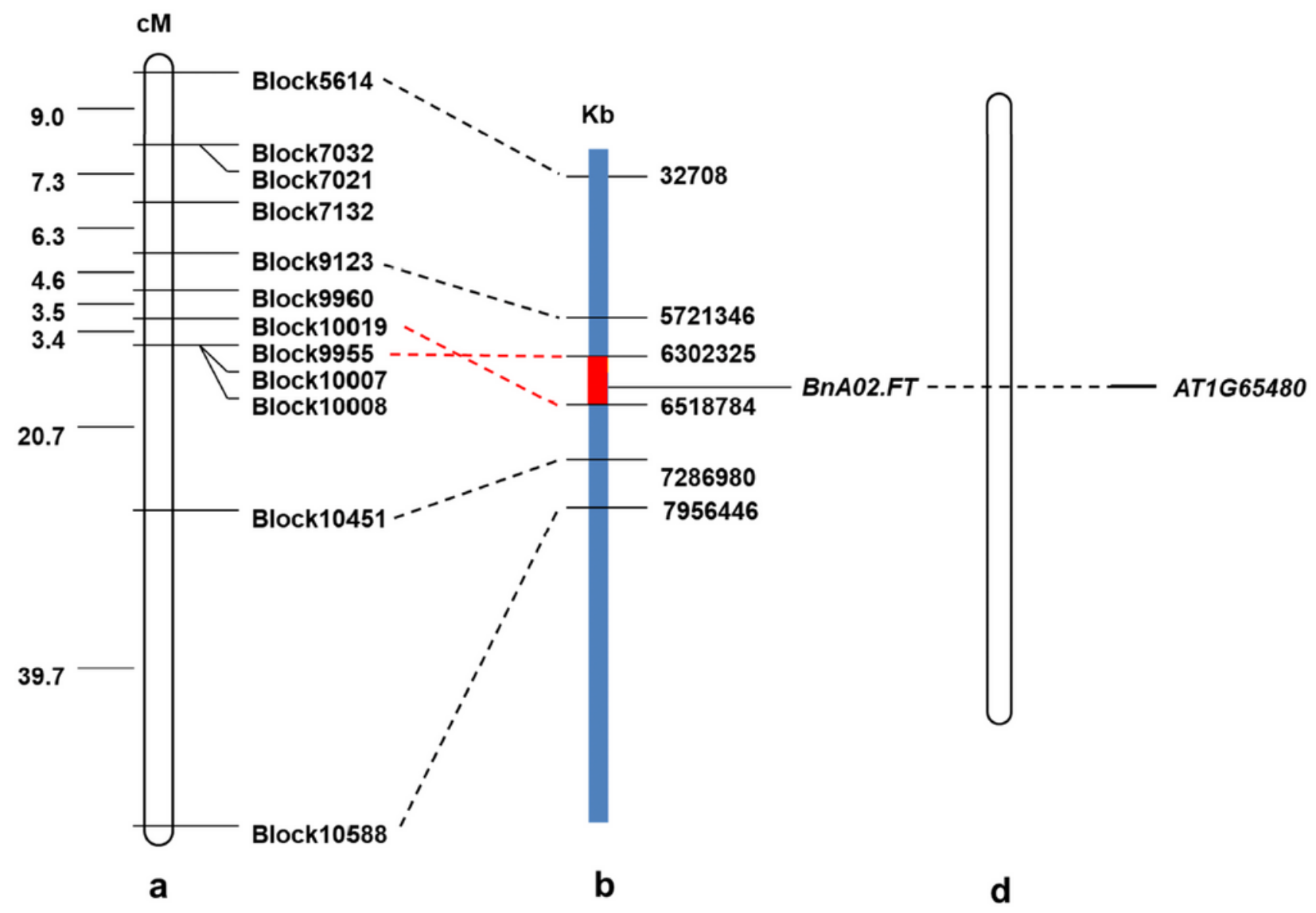

Figure 3

Analysis of candidate intervals for BnA02. The FT gene on chromosome A02 of B. napus was located by a combination of bulked segregant analysis and genetic linkage mapping. a a partial genetic linkage map of the area around the BnA02.FT gene, $b$ a partial physical map of linkage markers around the BnA02.FT gene. The red region indicates the sqFT-A02-4 candidate interval, corresponding to $6,302,325$ and $6,518,784 \mathrm{~kb}$, c results of a BLAST analysis in which the red target region was used against the Arabidopsis genome. The BnA02.FT gene is homologous to the AT1G65480 gene of A. thaliana.

\section{Supplementary Files}

This is a list of supplementary files associated with this preprint. Click to download.

- SupplementaryFiles.zip 